\begin{tabular}{|c|l|}
\hline Title & A berration-corrected multipole Wien filter for energy-filtered x-ray photoemi ssion electron microscopy \\
\hline Author(s) & Niimi, Hironobu; Chun, W ang-Jae; Suzuki, Shushi; A sakura, Kiy otaka; Kato, Makoto \\
\hline Citation & $\begin{array}{l}\text { Review of Scientific Instruments, 78(6), 063710 } \\
\text { https://doi.org/10.1063/2.2749449 }\end{array}$ \\
\hline Issue Date & 2007-06 \\
\hline Doc URL & http://hdl.handle.net/2115/25159 \\
\hline Rights & Copyright $\odot 2007$ A merican Institute of Physics \\
\hline Type & article \\
\hline File Information & RSI78-6.pdf \\
\hline
\end{tabular}

Instructions for use 


\title{
Aberration-corrected multipole Wien filter for energy-filtered x-ray photoemission electron microscopy
}

\author{
Hironobu Niimi, Wang-Jae Chun, Shushi Suzuki, and Kiyotaka Asakura ${ }^{a)}$ \\ Catalysis Research Center, Hokkaido University, 21-10 Kita, Kita-ku, Sapporo 001-0021, Japan and Core \\ Research for Evolutional Science and Technology, Japan Science and Technology Corporation, 21-10 Kita, \\ Kita-ku, Sapporo 001-0021, Japan \\ Makoto Kato \\ JEOL Ltd., 3-1-2 Musashino, Akishima, Tokyo 196-8558, Japan
}

(Received 17 April 2007; accepted 24 May 2007; published online 29 June 2007)

The aberration of a multipole Wien filter for energy-filtered x-ray photoemission electron microscopy was analyzed and the optimized Fourier components of the electric and magnetic fields for the third-order aperture aberration corrections were obtained. It was found that the third-order aperture aberration correction requires 12 electrodes and magnetic poles. () 2007 American Institute of Physics. [DOI: 10.1063/1.2749449]

\section{INTRODUCTION}

Surface inhomogeneity is an important factor in controlling surface chemical processes, such as catalysis. Such processes are often affected by interactions between inhomogeneous parts of the surface through diffusion over mesoscopic regions of a few tens of nanometer to micron order. As examples, oxygen diffusion in mixed oxides causes high selectivity for oxidation of hydrocarbons, and hydrogen migration from metal particles affects the acidity of supports. ${ }^{1-3}$ Another example is the hydrodesulfurization reaction on $\mathrm{Ni}_{2} \mathrm{P} / \mathrm{SiO}_{2}$ where partially formed $\mathrm{Ni}-\mathrm{S}$ bond plays an important role in $\mathrm{S}-\mathrm{C}$ bond scissions during the hydrodesulfurization. ${ }^{4,5}$ The characterization of spatial inhomogeneity in heterogeneous catalysts is a key issue in understanding these catalytic reactions.

Microscopic techniques are important for understanding these inhomogeneities. ${ }^{6}$ Recent developments in electron microscopes enable the in situ investigation of catalysis. ${ }^{7}$ However, electron damage in electron microscopes is large and may perturb the chemical reactions. Scanning probe microscopy [Scanning tunneling microscopy (STM) and atomic force microscopy (AFM)] can be used to investigate surfaces, though it gives little direct information about chemical distributions, such as elemental and chemical states. ${ }^{8-11}$

Photoemission electron microscopy (PEEM) is a promising tool for realizing chemical mapping in real time. ${ }^{12,13}$ It can provide information about surface spatiotemporal patterns, such as $\mathrm{CO}$ and $\mathrm{O}$ moving fronts and spiral and target patterns during $\mathrm{CO}$ oxidation processes. ${ }^{12}$ Contrast formation of the PEEM is realized through the work function change at the surface, and hence it is difficult to distinguish surface chemical species. When an energy-variable $x$-ray source is used, such as synchrotron radiation, the core electrons can be excited to become photoelectrons at x-ray energies corresponding to the absorption energies of the elements on the

${ }^{a)}$ Electronic mail: askr@cat.hokudai.ac.jp surface, which allows measurement of the elemental distribution on the sample. ${ }^{14,15}$ Since the number of emitted electrons is proportional to the $\mathrm{x}$-ray absorption coefficient, $\mathrm{x}$-ray absorption near edge structure ( $\mu$-XANES) can be obtained using PEEM and the chemical species on the surface can be identified. $^{16,17}$

In an alternative technique, a fixed x-ray energy source is used to eject photoelectrons from the sample and the kinetic energy of the electrons is measured, with chemical mapping of the surface performed in a similar way to x-ray photoelectron spectroscopy ( $\mu$-XPS) ${ }^{18-22}$ We call this technique energy-filtered $\mathrm{x}$-ray photoemission electron microscopy (EXPEEM) in this article. ${ }^{23,24}$ An advantage of EXPEEM is that synchrotron radiation need not be used. The measurement of the photoelectron energy can be achieved by using a hemispherical energy analyzer. ${ }^{18-20}$ However, in this case, the optical axis has to be bent in the hemispherical energy analyzer, which is not convenient for adjusting the optical axis and the lens condition. Thus, collinear arrangements of the lens and energy analyzer are desirable. There are several proposals for energy analyzers with a collinear arrangement, ${ }^{23-26}$ such as the Wien-filter-type energy analyzer shown in Fig. 1, where an electric field and a magnetic field are applied perpendicular to the path of the emitted electrons. In the filter, a moving electron feels a Lorentz force proportional to its velocity from the magnetic field, which is directed in an opposite direction to the electric field. The electron can only travel straight through the Wien filter when both forces are balanced. Several attempts have been made to obtain EXPEEM images using a Wien filter energy analyzer. $^{23,24,26}$ Yasuhuku et al. first reported an EXPEEM image of $\mathrm{Au}$ islands on a Ta sheet using the Wien filter energy analyzer. ${ }^{23}$

Another advantage of the Wien filter is that it can correct aberrations by adjusting the higher order Fourier components of the electric and magnetic fields. ${ }^{26-37}$ Rose has given the aberration collection condition (called the Rose condition in this article) to cancel out the second-order aberration by ad- 


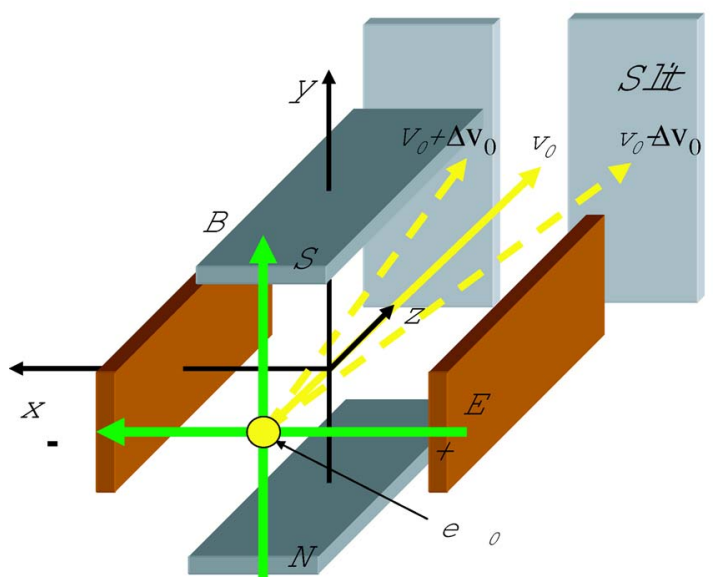

FIG. 1. Diagram of Wien filter.

justing the electric and magnetic fields up to the hexapole component. $^{32}$ We suggest another aberration correction (called as third-order aberration correction condition) that corrects up to third-order aperture aberration by adjusting the electric and magnetic fields up to the octupole component. ${ }^{33}$ This aberration correction can achieve a high energy resolution with a wide field of view.

In this article we first present details of the derivation of the aberration correction conditions of the Wien filter up to the third order. We then present the results of ray-tracing calculations and compare three conditions for minimizing aberration, including a new third-order aberration correction condition. Finally, we determine the optimum number of electrodes and magnetic poles for an effective Wien filter under the new third-order condition.

\section{OPTICS OF WIEN FILTER}

\section{A. Expansion of Lagrangian}

For mathematical simplicity, we use complex notation in this article,

$$
\begin{aligned}
& u=x+i y, \\
& \bar{u}=x-i y, \\
& |u|^{2}=x^{2}+y^{2}, \\
& \left|u^{\prime}\right|^{2}=x^{\prime 2}+y^{\prime 2}, \\
& \nabla_{u}=2 \frac{\partial}{\partial \bar{u}}=\frac{\partial}{\partial x}+i \frac{\partial}{\partial y}, \\
& \nabla_{u^{\prime}}=2 \frac{\partial}{\partial \bar{u}^{\prime}}=\frac{\partial}{\partial x^{\prime}}+i \frac{\partial}{\partial y^{\prime}} .
\end{aligned}
$$

Here $x^{\prime}, y^{\prime}$, and $u^{\prime}$ represent $d x / d z, d y / d z$, and $d u / d z$, respectively. $z$ is the electron optical axis. The Wien filter selects electrons with a specific kinetic energy using the balance condition between the eletrostatic and magnetic forces, as shown in Fig. 1.

We now describe the governing equation for the trajectory and aberration coefficients in the Wien filter as a function of the electric and magnetic fields. The electric and mag- netic fields in the Wien filter can be given by the general solution of the Laplace equation. ${ }^{38} \mathrm{We}$ assume that the electric and magnetic fields are uniform along the optical axis $(z$ direction) and the direction of the electric field is in the positive $x$ direction. The former assumption means that the fringe effect is neglected. This assumption allows us to derive the aberration coefficients in explicit forms and optimize them analytically. As shown in a previous paper, ${ }^{28}$ the fringe effect is negligibly small as long as the distributions of the electric and magnetic fields coincide. A following paper will discuss how this condition is practically satisfied in the Wien filter. ${ }^{39}$ The assumption that the electric field points in the $x$ direction can be expressed as a real electrostatic potential $\Phi(u, \bar{u}),{ }^{40}$

$$
\Phi(u, \bar{u})=-\sum_{m=0}^{\infty} \phi_{m c} \frac{u^{m}+\bar{u}^{m}}{2},
$$

where $\phi_{m c}$ is the $m$ th order coefficient for the electric field. $-\phi_{0 c}$ is equal to the pass energy $\phi_{0}=m_{0} v_{0}^{2} / 2$. Likewise, the magnetic scalar potential $\Psi(u, \bar{u})$ can be written under the assumption that the magnetic field is applied perpendicular to the electric field, which requires that the magnetic scalar potential $\Psi$ be imaginary,

$$
\mu_{0} \Psi(u, \bar{u})=-\sum_{m=0}^{\infty} \psi_{m s} \frac{u^{m}-\bar{u}^{m}}{2 i},
$$

where $\psi_{m s}$ is the $m$ th order coefficient for the magnetic field. In Cartesian coordinates, $\Phi(u, \bar{u})$ and $\mu_{0} \Psi(u, \bar{u})$ are written as

$$
\begin{gathered}
\Phi(x, y)=\phi_{0}-\phi_{1 c} x-\phi_{2 c}\left(x^{2}-y^{2}\right)-\phi_{3 c} x\left(x^{2}-3 y^{2}\right) \\
-\phi_{4 c}\left(x^{4}-6 x^{2} y^{2}-y^{4}\right)-\cdots, \\
\mu_{0} \Psi(x, y)=-\psi_{1 s} y-2 \psi_{2 s} x y-\psi_{3 s} y\left(3 x^{2}-y^{2}\right) \\
-4 \psi_{4 s} x y\left(x^{2}-y^{2}\right)-\cdots,
\end{gathered}
$$

where $\phi_{0 c}, \phi_{1 c}, \phi_{2 c}, \ldots$ and $\psi_{1 s}, \psi_{2 s}, \psi_{3 s}, \ldots$ are the coefficients of the Fourier expansions of the electric and magnetic fields. The motion of the electron in the Wien filter is determined by the following Lagrangian: ${ }^{38}$

$$
F\left(u, \bar{u}, u^{\prime}, \bar{u}^{\prime}\right)=\sqrt{\Phi(u, \bar{u})\left(1+\left|u^{\prime}\right|^{2}\right)}-\sqrt{\frac{e}{2 m_{0}}} A_{z}(u, \bar{u}) .
$$

Using the relation $\boldsymbol{B}=\boldsymbol{\nabla} \times \boldsymbol{A}=-\mu_{0} \boldsymbol{\nabla} \Psi$ gives $^{41}$

$$
A_{z}(u, \bar{u})=-\sum_{m=0}^{\infty} \psi_{m s} \frac{u^{m}+\bar{u}^{m}}{2} .
$$

In order to solve the Euler-Lagrange equation

$$
\frac{d}{d z}\left(\nabla_{u^{\prime}} F\right)-\nabla_{u} F=0
$$

for each order of $u$, the Lagrangian $F$ in (11) is expanded in a power series of $u, \bar{u}, u^{\prime}$, and $\bar{u}^{\prime}$ up to fourth order,

$$
F=F_{0}+F_{1}+F_{2}+F_{3}+F_{4} \text {. }
$$

This expansion has one further step than the calculation given by Rose. ${ }^{32}$ Accordingly, $F_{1}, F_{2}, F_{3}$, and $F_{4}$ are represented by 


$$
\begin{aligned}
F_{1}= & -\frac{1}{4 \phi_{0}^{1 / 2}}\left(\phi_{1 c}-v_{0} \psi_{1 s}\right)(u+\bar{u}) \\
F_{2}= & -\frac{1}{32 \phi_{0}^{3 / 2}}\left[\phi_{1 c}^{2}(u+\bar{u})^{2}-16 \phi_{0}^{2}\left|u^{\prime}\right|^{2} 8 \phi_{0}\left(\phi_{2 c}-v_{0} \psi_{2 s}\right)\right. \\
& \left.\times\left(u^{2}+\bar{u}^{2}\right)\right]
\end{aligned}
$$

$$
\begin{aligned}
F_{3}= & -\frac{1}{128 \phi_{0}^{5 / 2}}\left[\phi_{1 c}^{3}(u+\bar{u})^{3}+8 \phi_{0} \phi_{1 c} \phi_{2 c}(u+\bar{u})\left(u^{2}+\bar{u}^{2}\right)\right. \\
& -16 \phi_{0}^{2} \phi_{1 c}(u+\bar{u})\left|u^{\prime}\right|^{2}+32 \phi_{0}^{2}\left(\phi_{3 c}-v_{0} \psi_{3 s}\right)\left(u^{3}\right. \\
& \left.\left.+\bar{u}^{3}\right)\right],
\end{aligned}
$$

$$
\begin{aligned}
F_{4}= & -\frac{1}{2048 \phi_{0}^{7 / 2}}\left[-5 \phi_{1 c}^{4}(u+\bar{u})^{4}+48 \phi_{0} \phi_{1 c}^{2} \phi_{2 c}(u+\bar{u})^{2}\left(u^{2}+\bar{u}^{2}\right)+64 \phi_{0}^{2} \phi_{2 c}^{2}\left(u^{2}+\bar{u}^{2}\right)+128 \phi_{0} \phi_{1 c} \phi_{3 c}(u+\bar{u})\left(u^{3}+\bar{u}^{3}\right)\right. \\
& \left.+32 \phi_{0}^{2} \phi_{1 c}^{2}(u+\bar{u})^{2}\left|u^{\prime}\right|^{2}+256 \phi_{0}^{3} \phi_{2 c}\left(u^{2}+\bar{u}^{2}\right)\left|u^{\prime}\right|^{2}+256 \phi_{0}^{4}\left|u^{\prime}\right|^{4}+512 \phi_{0}^{3}\left(\phi_{4 c}-v_{0} \psi_{4 s}\right)\left(u^{4}+\bar{u}^{4}\right)\right] .
\end{aligned}
$$

In Cartesian coordinates we have

$$
\begin{aligned}
F_{1}= & -\frac{1}{4 \phi_{0}^{1 / 2}}\left(\phi_{1 c}-v_{0} \psi_{1 s}\right) 2 x, \\
F_{2}= & -\frac{1}{32 \phi_{0}^{3 / 2}}\left[\phi_{1 c}^{2}\left(4 x^{2}\right)-16 \phi_{0}^{2}\left(x^{\prime 2}+y^{\prime 2}\right)+16 \phi_{0}\left(\phi_{2 c}\right.\right. \\
& \left.\left.-v_{0} \psi_{2 s}\right)\left(x^{2}-y^{2}\right)\right],
\end{aligned}
$$

$$
\begin{aligned}
F_{3}= & -\frac{1}{128 \phi_{0}^{5 / 2}}\left[\phi_{1 c}^{3}\left(8 x^{3}\right)+8 \phi_{0} \phi_{1 c} \phi_{2 c} 4 x\left(x^{2}-y^{2}\right)\right. \\
& -32 \phi_{0}^{2} \phi_{1 c} x\left(x^{\prime 2}+y^{\prime 2}\right)+128 \phi_{0}^{2}\left(\phi_{3 c}-v_{0} \psi_{3 s}\right)\left(x^{3}\right. \\
& \left.\left.-3 x y^{2}\right)\right],
\end{aligned}
$$

$$
\begin{aligned}
F_{4}= & -\frac{1}{2048 \phi_{0}^{7 / 2}}\left[5 \phi_{1 c}^{4}\left(16 x^{4}\right)+384 \phi_{0} \phi_{1 c}^{2} \phi_{2 c} x^{2}\left(x^{2}-y^{2}\right)+128 \phi_{0}^{2} \phi_{2 c}^{2}\left(x^{2}-y^{2}\right)+512 \phi_{0} \phi_{1 c} \phi_{3 c} x\left(x^{3}-3 x y^{2}\right)+128 \phi_{0}^{2} \phi_{1 c}^{2} x^{2}\left(x^{\prime 2}\right.\right. \\
& \left.\left.+y^{\prime 2}\right)+256 \phi_{0}^{3} \phi_{2 c}\left(x^{2}-y^{2}\right)\left(x^{\prime 2}+y^{\prime 2}\right)+256 \phi_{0}^{4}\left(x^{\prime 2}+y^{\prime 2}\right)^{2}+1024 \phi_{0}^{3}\left(\phi_{4 c}-v_{0} \psi_{4 s}\right)\left(x^{4}-6 x^{2} y^{2}+y^{4}\right)\right] .
\end{aligned}
$$

By solving the Euler-Lagrange equation, trajetory $u, u$ $=u_{1}+\Delta u_{2}+\Delta u_{3}+\cdots$, is obtained, where $u_{1}, \Delta u_{2}$, and $\Delta u_{3}$ are the solutions of the first-order trajectory, the second-order aberration, and the third-order aberration, respectively.

\section{B. First-order trajectory equation}

To derive the first-order trajectory, we use the Lagrangian up to the second-order power series $\left(F=F_{0}+F_{1}+F_{2}\right)$ from expression (13),

$$
\begin{aligned}
u_{1}^{\prime \prime}+ & \frac{1}{\phi_{0}}\left[\frac{\phi_{1 c}^{2}}{8 \phi_{0}}\left(u_{1}+\overline{u_{1}}\right)+\left(\phi_{2 c}-v_{0} \psi_{2 s}\right) \overline{u_{1}}\right]=-\frac{1}{2 \phi_{0}}\left(\phi_{1 c}\right. \\
& \left.-v_{0} \psi_{1 s}\right) .
\end{aligned}
$$

The right-hand side of expression (23) corresponds to the term of the deflection force for the electron. The balance condition to cancel out this deflection force, namely, the Wien condition, is given by

$$
\phi_{1 c}-v_{0} \psi_{1 s}=0 .
$$

The Wien filter requires the stigmatic condition that the trajectory of the $x$ direction coincides with that of the $y$ direc- tion at the exit focal plane. Since the real and imaginary parts of expression (23) correspond to the $x$ and $y$ directions, respectively, the stigmatic condition is satisfied when the real part of the second term of expression (23) is equal to the imaginary part. This condition is expressed as

$$
\phi_{2 c}-v_{0} \psi_{2 s}=-\frac{1}{8 \phi_{0}} \phi_{1 c}^{2} .
$$

For convenience, the normalized Fourier components of electric and magnetic fields, $e_{2}-e_{4}$ and $b_{2}-b_{4}{ }^{42}$ are introduced to describe the following results:

$$
\begin{array}{ll}
e_{2}=\frac{\phi_{2 c}}{\phi_{1 c}} R_{0}, & b_{2}=\frac{\psi_{2 s}}{\psi_{1 s}} R_{0}, \\
e_{3}=\frac{\phi_{3 c}}{\phi_{1 c}} R_{0}^{2}, & b_{3}=\frac{\psi_{3 s}}{\psi_{1 s}} R_{0}^{2}, \\
e_{4}=\frac{\phi_{4 c}}{\phi_{1 c}} R_{0}^{3}, & b_{4}=\frac{\psi_{4 s}}{\psi_{1 s}} R_{0}^{3},
\end{array}
$$


where $R_{0}=2 \phi_{0} / \phi_{1}$.

Consequently, expression (25) can be described as

$$
e_{2}-b_{2}=-\frac{1}{4}
$$

In addition, second-order aberration was reduced by designing the shape of the electromagnetic pole structure according to the calculations of Kato and Tsuno. ${ }^{28}$ This optimizing condition is called the orthogonal condition and is satisfied in our previously developed Wien filter EXPEEM system. $^{23}$

\section{Derivation of higher-order aberration coefficients in a uniform field approximation}

\section{Second-order aberrations}

Since aperture aberration of the filter affects the isochromatic image, ${ }^{32}$ it must be reduced to achieve a high resolution Wien filter with high sensitivity. We derive the aberration coefficients of the Wien filter using the third-order Lagrangian $\left(F=F_{0}+F_{1}+F_{2}+F_{3}\right)$ under the Wien and stigmatic conditions

$$
\Delta u_{2}^{\prime \prime}+\frac{\phi_{1 c}^{2}}{8 \phi_{0}^{2}} \Delta u_{2}=\frac{1}{\phi_{0}^{1 / 2}}\left[\nabla_{u} F_{3}-\left(\nabla_{u^{\prime}} F_{3}\right)^{\prime}\right] .
$$

The second-order aberrations, $\Delta u_{2}(z)$, and the slope, $\Delta u_{2}^{\prime}(z)$, can be obtained at the imaging plane as follows:

$$
\begin{aligned}
& \Delta u_{2}\left(z_{i}\right)=\frac{1}{\phi_{0}^{1 / 2}} \int_{z_{0}}^{z_{i}}\left(\Delta_{u_{0}^{\prime}} F_{3}^{\mathrm{int}}\right) d z, \\
& \Delta u_{2}^{\prime}\left(z_{i}\right)=\frac{1}{\phi_{0}^{1 / 2}}\left[\left[\Delta_{u_{0}^{\prime}} F_{3}^{\mathrm{int}}\right]_{z_{0}}^{z_{i}}-\int_{z_{0}}^{z_{i}}\left(\Delta_{u_{0}} F_{3}^{\mathrm{int}}\right) d z\right],
\end{aligned}
$$

where $z_{0}$ and $z_{i}$ represent the entrance and image positions, respectively. $F_{3}^{\text {int }}$ is the third-order Lagrangian in the interaction picture defined as follows:

$$
\begin{aligned}
F_{3}^{\text {int }}\left(u_{0}, \bar{u}_{0}, u_{0}^{\prime}, \bar{u}_{0}^{\prime} ; z\right)= & F_{3}\left[u_{0} g(z)+u_{0}^{\prime} h(z), \overline{u_{0}} g(z)\right. \\
& +\bar{u}_{0}^{\prime} h(z), u_{0} g^{\prime}(z)+u_{0}^{\prime} h_{0}^{\prime}(z), \bar{u}_{0} g^{\prime}(z) \\
& \left.+\bar{u}_{0}^{\prime} h^{\prime}(z)\right],
\end{aligned}
$$

where $g(z)$ and $h(z)$ are two fundamental solutions of (23) with initial conditions $g\left(z_{0}\right)=1, g^{\prime}\left(z_{0}\right)=0, h\left(z_{0}\right)=0$, and $h^{\prime}\left(z_{0}\right)=1$. $g(z)$ and $h(z)$, together with $g^{\prime}(z)$ and $h^{\prime}(z)$, form the first-order transfer matrix $S$,

$$
S=\left(\begin{array}{cc}
g(z) & h(z) \\
g^{\prime}(z) & h^{\prime}(z)
\end{array}\right) .
$$

Thus, $F_{3}^{\text {int }}(z)$ is the $F_{3}$ value on the first-order trajectory at the general position $z . F_{3}^{\text {int }}(z)$ represents the pure effect of the perturbation and can change only with the perturbation. ${ }^{33}$ Analytical integrations of (29) give the relation between the aberration coefficients $C$ (pqrs) defined by the following equation:

$$
\Delta u_{n}=\sum_{p+q+r+s=n} C(p q r s) u_{o}^{p} \bar{u}_{o}^{q} u_{o}^{\prime r} \bar{u}_{o}^{\prime s},
$$

where $\Delta u_{n}$ is the $n$th order aberration. The second-order aperture aberration $C_{2}$ (pqrs) is expressed by the Fourier com- ponents of the electric and magnetic fields as follows:

$$
\begin{aligned}
& C_{2}(2000)=-\frac{2}{3 R_{0}} b_{2}, \\
& C_{2}(1100)=-\frac{1}{3 R_{0}}\left(3+4 b_{2}\right), \\
& C_{2}(0200)=-\frac{2}{R_{0}}\left[b_{2}+2\left(e_{3}-b_{3}\right)\right], \\
& C_{2}(0020)=-\frac{2 R_{0}}{3}\left(3+4 b_{2}\right), \\
& C_{2}(0011)=-\frac{4 R_{0}}{3}\left(3+4 b_{2}\right), \\
& C_{2}(0002)=-8 R_{0}\left[b_{2}+2\left(e_{3}-b_{3}\right)\right] .
\end{aligned}
$$

To cancel out the second-order aberration $\left(\Delta u_{n}=0\right)$, $C($ pqrs $)=0(p+q+r+s \leqslant 2)$ must be satisfied. We assume a point source at the entrance $\left(u_{0}=\bar{u}_{0}=0\right)$. Consequently, the coefficients with $p \neq 0$ and $q \neq 0$ can be neglected and we obtain the following condition for the second-order aberrations:

$$
e_{2}=-1, \quad b_{2}=-3 / 4, \quad e_{3}-b_{3}=3 / 8 .
$$

The condition agrees with that given by Rose. ${ }^{32}$ We call this condition the Rose condition.

The following section presents a new third-order aberration correction condition.

\section{Third-order aperture aberrations}

We can similarly determine the third-order aberration coefficients $C_{3}$ (pqrs) using the Lagrangian up to fourth order $\left(F=F_{0}+F_{1}+F_{2}+F_{3}+F_{4}\right)$. The third-order aberration $\Delta u_{3}$ is given by the following equation:

$$
\begin{aligned}
\Delta u_{3}^{\prime \prime}+\frac{\phi_{1 c}^{2}}{8 \phi_{0}^{2}} \Delta u_{3}= & \frac{1}{\phi_{0}^{1 / 2}}\left[D_{2}\left(\nabla_{u} F_{3}\right)-\left[D_{2}\left(\nabla_{u^{\prime}} F_{3}\right)\right]^{\prime}+\nabla_{u} F_{4}\right. \\
& \left.-\left(\nabla_{u^{\prime}} F_{4}\right)^{\prime}\right]
\end{aligned}
$$

where

$$
D_{2}=\Delta u_{2} \frac{\partial}{\partial u}+\Delta \bar{u}_{2} \frac{\partial}{\partial \bar{u}}+\Delta u_{2}^{\prime} \frac{\partial}{\partial u^{\prime}}+\Delta \bar{u}_{2}^{\prime} \frac{\partial}{\partial \bar{u}^{\prime}} .
$$

Since the equation in the left side is linear, the solution can be divided into two parts; one $\left(\Delta u_{3 A}\right)$ related to $F_{3}$ and the other $\left(\Delta u_{3 B}\right)$ related to $F_{4}$,

$$
\begin{aligned}
& \Delta u_{3 A}^{\prime \prime}+\frac{\phi_{1 c}^{2}}{8 \phi_{0}^{2}} \Delta u_{3 A}=\frac{1}{\phi_{0}^{1 / 2}}\left[D_{2}\left(\nabla_{u} F_{3}\right)-\left[D_{2}\left(\nabla_{u^{\prime}} F_{3}\right)\right]^{\prime}\right], \\
& \Delta u_{3 B}^{\prime \prime}+\frac{\phi_{1 c}^{2}}{8 \phi_{0}^{2}} \Delta u_{3 B}=\frac{1}{\phi_{0}^{1 / 2}}\left[\nabla_{u} F_{4}-\left(\nabla_{u^{\prime}} F_{4}\right)^{\prime}\right] .
\end{aligned}
$$

Both parts can be independently solved at the imaging plane, $z=z_{i}$ 

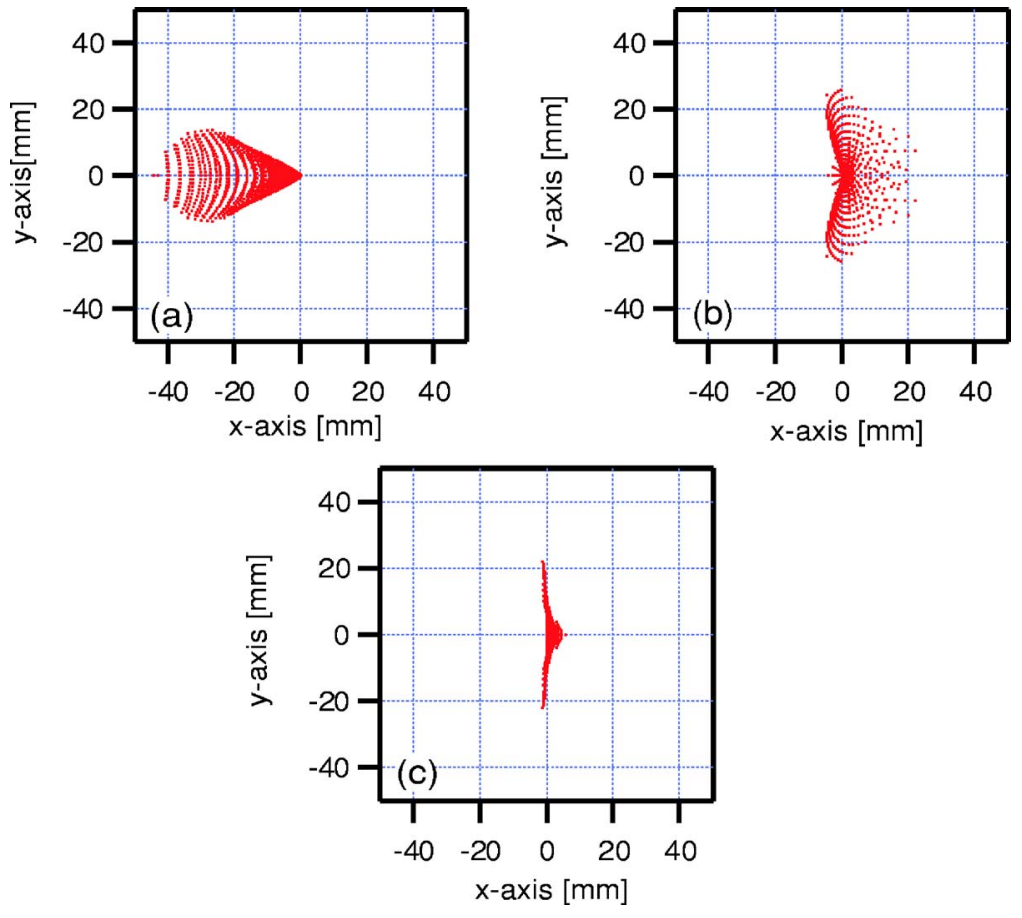

$$
\begin{aligned}
& \Delta u_{3 A}\left(z_{i}\right)=\frac{1}{\phi_{0}^{1 / 2}} \int_{z_{0}}^{z_{i}} D_{2}^{\mathrm{int}}\left(\nabla_{u_{0}^{\prime}} F_{3}^{\mathrm{int}}\right) d z, \\
& \Delta u_{3 B}\left(z_{i}\right)=\frac{1}{\phi_{0}^{1 / 2}} \int_{z_{0}}^{z_{i}}\left(\nabla_{u_{0}^{\prime}} F_{4}^{\mathrm{int}}\right) d z .
\end{aligned}
$$

The differential operator $D_{2}^{\text {int }}$ is given by

$$
\begin{aligned}
D_{2}^{\text {int }}= & \Delta u_{2}^{\text {int }}(z) \frac{\partial}{\partial u_{0}}+\Delta \bar{u}_{2}^{\text {int }}(z) \frac{\partial}{\partial \bar{u}_{0}}+\Delta u_{2}^{\prime \text { int }}(z) \frac{\partial}{\partial u_{0}^{\prime}} \\
& +\Delta \bar{u}_{2}^{\prime \text { int }}(z) \frac{\partial}{\partial \bar{u}_{0}^{\prime}} .
\end{aligned}
$$

Finally, Eq. (37) is integrated and the third-order aperture aberration $C_{3}$ (pqrs) or the aberration for a point source ( $p$ $=q=0)$ is obtained as follows: ${ }^{33}$

$$
\begin{aligned}
C_{3}(0030)= & \frac{\pi R_{0}}{24 \sqrt{2}}\left(3+4 b_{2}\right)\left[3+40 b_{2}+60\left(e_{3}-b_{3}\right)\right] \\
& -\frac{\pi R_{0}}{8 \sqrt{2}}\left(3+20 b_{2}+12 e_{3}\right),
\end{aligned}
$$

TABLE I. Aberration correction coefficients. (A) Orthogonal condition, (B) Rose condition, and (C) third-order aberration correction condition.

\begin{tabular}{llll}
\hline \hline & $\mathrm{A}$ & $\mathrm{B}$ & $\mathrm{C}$ \\
\hline$e_{2}$ & -0.250 & -1.000 & -1.000 \\
$b_{2}$ & 0 & -0.750 & -0.750 \\
$e_{3}$ & 0 & -0.375 & 0.563 \\
$b_{3}$ & 0 & 0 & 0.188 \\
$e_{4}$ & 0 & 0 & -0.180 \\
$b_{4}$ & 0 & 0 & 0 \\
\hline \hline
\end{tabular}

FIG. 2. Comparison of aberrations given in Table I (a) condition A, (b) condition B, and (c) condition C. The horizontal direction corresponds to the energy dispersion direction.

$$
\begin{aligned}
C_{3}(0021)= & \frac{\pi R_{0}}{6 \sqrt{2}}\left[21+66 b_{2}+140 b_{2}^{2}+360 b_{2}\left(e_{3}-b_{3}\right)\right. \\
& \left.+360\left(e_{3}-b_{3}\right)^{2}\right]-\frac{\pi R_{0}}{2 \sqrt{2}}\left(5+6 b_{2}+6 b_{2}^{2}\right), \\
C_{3}(0012)= & \frac{\pi R_{0}}{8 \sqrt{2}}\left(3+4 b_{2}\right)\left[3+40 b_{2}+60\left(e_{3}-b_{3}\right)\right] \\
& -\frac{3 \pi R_{0}}{8 \sqrt{2}}\left(3+20 b_{2}+12 e_{3}\right), \\
C_{3}(0003)= & \frac{\pi R_{0}}{\sqrt{2}}\left(3+10 b_{2}\right)\left[b_{2}+2\left(e_{3}-b_{3}\right)\right]-\frac{3 \pi R_{0}}{\sqrt{2}}\left[b_{2}\right. \\
& \left.+b_{2}^{2}+2 e_{3}+4\left(e_{4}-b_{4}\right)\right] .
\end{aligned}
$$

Under the Rose condition, it is difficult to optimize the above four equations. Thus we eliminate the aberration in the energy dispersion direction, i.e., the $x$ direction corresponding to the real part of $\Delta u_{3}$,

$$
\begin{aligned}
\Delta x_{3}= & \frac{1}{2}\left(\Delta u_{3}+\Delta \bar{u}_{3}\right)=C_{3}(0030)\left(x^{\prime 3}-3 x^{\prime} y^{\prime 2}\right) \\
& +C_{3}(0021)\left(x^{\prime 3}+x^{\prime} y^{\prime 2}\right)+C_{3}(0012)\left(x^{\prime 3}+x^{\prime} y^{\prime 2}\right) \\
& +C_{3}(0003)\left(x^{\prime 3}-3 x^{\prime} y^{\prime 2}\right)=\left[C_{3}(0030)+C_{3}(0021)\right. \\
& \left.+C_{3}(0012)+C_{3}(0003)\right] x^{\prime 3}+\left[-3 C_{3}(0030)\right. \\
& \left.+C_{3}(0021)+C_{3}(0012)-3 C_{3}(0003)\right] x^{\prime} y^{\prime 2}
\end{aligned}
$$

$\Delta x_{3}$ is zero when the coefficients of $x^{\prime 3}$ and $x^{\prime} y^{\prime 2}$ are zero. This condition is derived as follows:

$$
\begin{aligned}
& e_{2}=-1, \quad b_{2}=-3 / 4, \quad e_{3}=0.563, \\
& b_{3}=0.188, \quad e_{4}-b_{4}=-0.180 .
\end{aligned}
$$

We have summarized three conditions in Table I, namely, the 


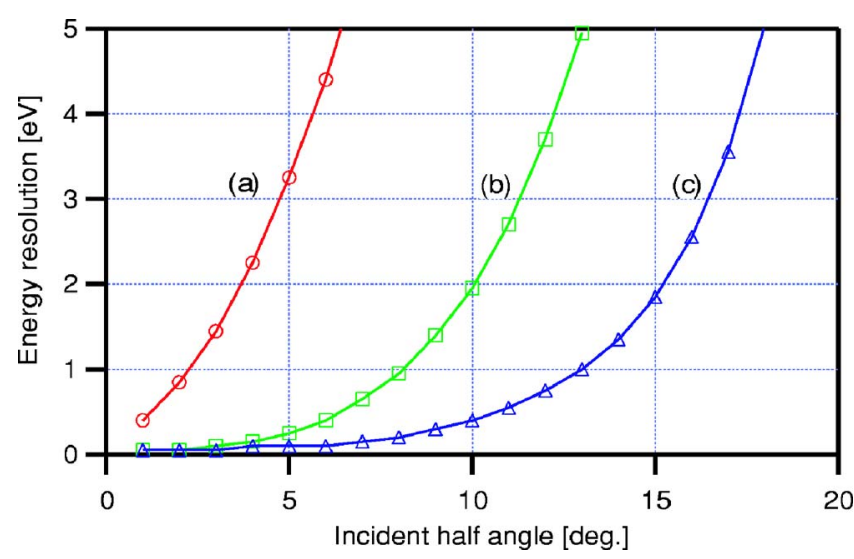

FIG. 3. Dependence of the energy resolution on the incident half angle: (a) condition $\mathrm{A},(\mathrm{b})$ condition $\mathrm{B}$, and (c) condition $\mathrm{C}$.

orthogonal condition (A), Rose condition (B), and thirdorder aberration correction condition $(\mathrm{C})$.

In the next section, we present the ray-tracing results to demonstrate how these conditions work.

\section{RAY TRACING}

We compare the aberration for the three conditions in Table I. We performed ray tracing using ideal electric and magnetic fields. The conditions used in calculating the aberration are shown in Table II, where $E_{p}, L, \alpha_{0 \max }$, and $\left(x_{o}, y_{o}\right)$ are defined as follows:

$E_{p}$ is a pass energy of analyzer that allows the electron transmission with corresponding kinetic energy, $L$ is a filter length which corresponds to the length between entrance and exit slits, $\alpha_{0 \max }$ is a maximum incident angle of the electron, and $\left(x_{o}, y_{o}\right)$ is an incident position of the electron.
TABLE II. Conditions for calculation of the aberration results in Fig. 3.

\begin{tabular}{llll}
\hline \hline$E_{p}{ }^{\mathrm{a}}(\mathrm{eV})$ & $L^{\mathrm{b}}(\mathrm{mm})$ & $\alpha_{0 \max }{ }^{\mathrm{c}}(\mathrm{deg})$ & $\left(x_{o}, y_{o}\right)^{\mathrm{d}}(\mathrm{mm})$ \\
\hline 100 & 400 & 14 & $(0,0)$ \\
\hline
\end{tabular}

${ }^{\mathrm{a}} \mathrm{A}$ pass energy of analyzer that allows the electron transmission with corresponding kinetic energy.

${ }^{b}$ A filter length which corresponds to the length between entrance and exit slits.

${ }^{\mathrm{c}}$ A maximum incident angle of the electron.

${ }^{\mathrm{d}}$ An incident position of the electron.

The energy dispersion direction, namely, the deflection direction of an electron, is taken to be the $x$ direction. The aberration for conditions $\mathrm{A}, \mathrm{B}$, and $\mathrm{C}$ are shown in Figs. 2(a)-2(c), respectively. The blurs of the energy dispersion direction for each aberration are about 27, 12, and $3 \mathrm{~mm}$ for conditions $\mathrm{A}, \mathrm{B}$, and $\mathrm{C}$, respectively, indicating that the aberration is most effectively reduced for condition $\mathrm{C}$ in the energy dispersion direction.

Figure 3 shows the dependence of the energy resolution on the incident half angle $\alpha$. For a $1 \mathrm{eV}$ energy resolution, $\alpha$ should be $2^{\circ}, 8^{\circ}$ and $13^{\circ}$ for conditions $\mathrm{A}, \mathrm{B}$, and $\mathrm{C}$, respectively. If the intensity of the final image $I$ is determined by the incident half angle, the image intensity for condition $\mathrm{C}$ is 42 times larger than that of condition A. For an energy resolution of $0.4 \mathrm{eV}, \alpha$ should be $1^{\circ}, 6^{\circ}$, and $10^{\circ}$ for conditions $\mathrm{A}, \mathrm{B}$, and $\mathrm{C}$, respectively. In this case, the image intensity of condition $\mathrm{C}$ is 100 times larger than that of condition A.

\section{NUMBER OF ELECTRODES AND MAGNETIC POLES}

A multipole Wien filter can produce fields satisfying condition $C .^{26,28,32}$ However, it must be determined how
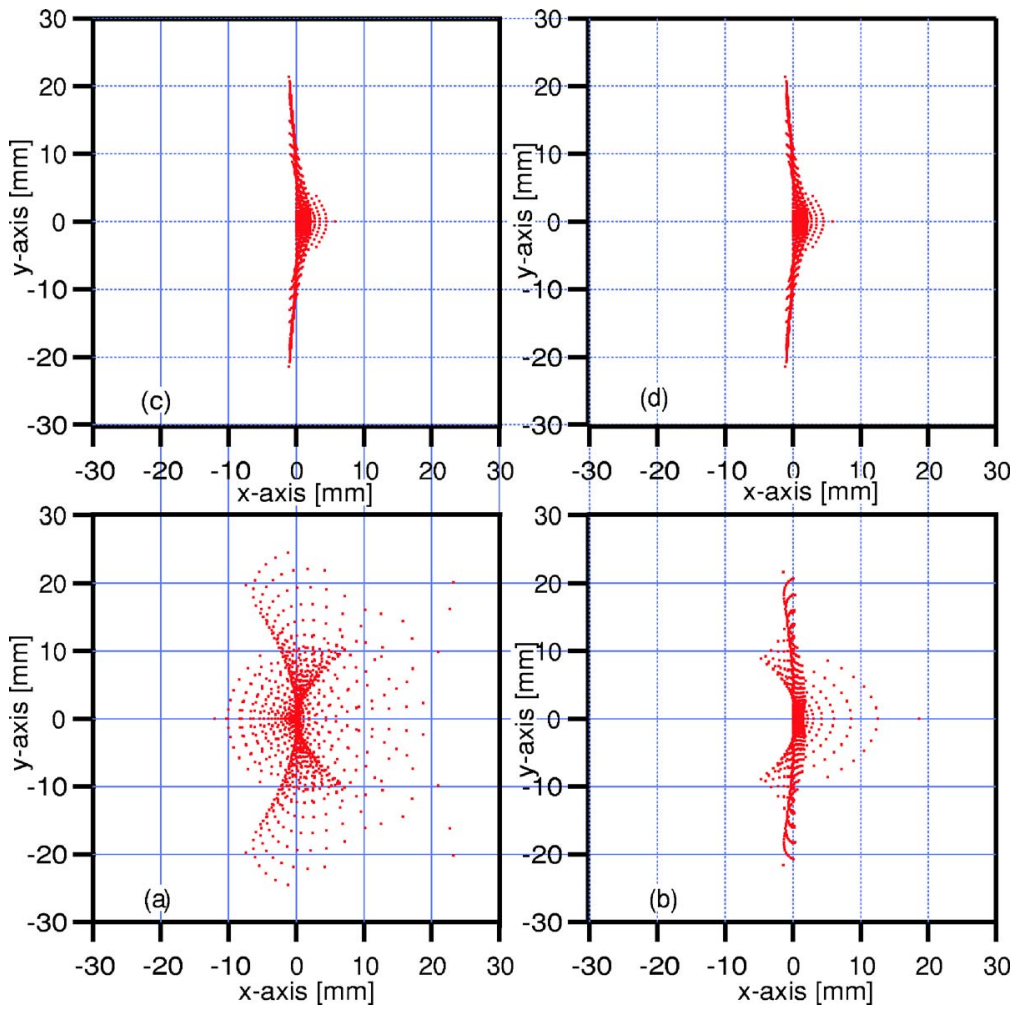

FIG. 4. Comparison of the aberration for (a) 8 electrodes and magnetic poles, (b) 10 electrodes and magnetic poles, (c) 12 electrodes and magnetic poles, and (d) 18 electrodes and magnetic poles. The horizontal direction corresponds to the energy dispersion direction. 

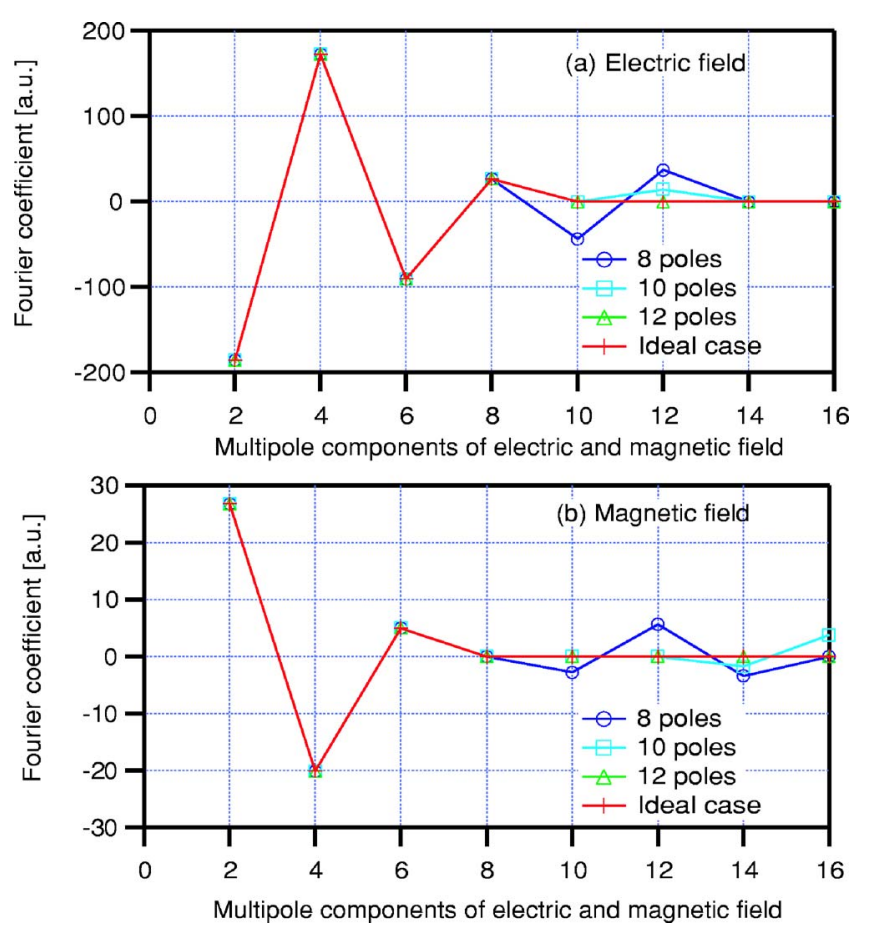

FIG. 5. $n$th Fourier components of (a) electric and (b) magnetic fields. The circles, triangles, squares, and crosses correspond to the cases of 8,10 , and 12 electrodes and magnetic poles and the ideal condition, respectively.

many poles are required. The parameters $e_{4}$ and $b_{4}$ in condition $\mathrm{C}$ must be controlled, and hence octupoles are necessary, requiring at least eight electrodes and magnetic poles. We calculated the fields satisfying condition $\mathrm{C}$ using the charge simulation method (CSM), ${ }^{38,43}$ where virtual electric and magnetic charges are arranged on the pole surfaces to make a potential satisfying condition $\mathrm{C}$. We then calculated the electron trajectory, including the fringe effect. Figure 4(a) shows the aberration for eight electrodes and magnetic poles. The blurring of the aberration is about $30 \mathrm{~mm}$. The reason that the aberration is so large is that Fourier components of Eqs. (9) and (10) higher than fourth order cannot be controlled, as shown in Fig. 5, which causes deterioration of the aberration. In order to reduce the higher order Fourier components, we added further poles and calculated the aberration. Twelve poles gave a sufficiently small dispersion in the $x$ direction, as shown in Fig. 4(c). We further increased the number of electrodes and magnetic poles to 18 , but the aberration was little improved, as shown in Fig. 4(d). Thus, 12 electrodes and magnetic poles are practically the best choice for the multipole Wien filter.

In a following paper we discuss the design of the Wien filter and possibilities for laboratory EXPEEM.

\section{DISCUSSIONS}

In this article, we have investigated a Wien filter with small aberration and large filter size, considered a promising candidate for the development of a high brilliance and high energy resolution filter. In order to obtain the aberration conditions explicitly, we expressed the aberration coefficients up to third order using normalized coefficients of the multipole expansion formula for electric and magnetic fields under the uniform field approximation. By monitoring the electron trajectory, we found that the incident angle can be increased while maintaining a high energy resolution. We have determined using CSM that the optimal number of electrodes and magnetic poles of the multipole Wien filter is 12. Accordingly, we can provide a basic design for a multipole Wien filter with less aberration, which will be important in developing an imaging energy analyzer with a straight optical axis and a bright, high resolution filter.

\section{ACKNOWLEDGMENTS}

This research is supported by a Grant-in-Aid for Scientific Research Category S No. 16106010 from JSPS, a Grant for Joint Project between University and Industry "EXPEEM" from MEXT and CREST of JST.

${ }^{1}$ B. Delmon and G. F. Froment, Catal. Rev. - Sci. Eng. 38, 69 (1996).

${ }^{2}$ Y. Ohminami et al., Bull. Chem. Soc. Jpn. 78, 435 (2005).

${ }^{3}$ New Aspects of Spillover Effect in Catalysis: for Development of Highly Active Catalysts, edited by T. Inui, K. Fujimoto, T. Uchijima, and M. Masai (Elsevier, Amsterdam, 1993).

${ }^{4}$ S. T. Oyama, J. Catal. 216, 343 (2003).

${ }^{5}$ T. Kawai, K. K. Bando, Y. K. Lee, S. T. Oyama, W. J. Chun, and K. Asakura, J. Catal. 241, 20 (2006).

${ }^{6}$ N. Yao and Z. L. Wang, Handbook of Microscopy for Nanotechnology (Kluwer, Boston, 2005).

${ }^{7}$ P. L. Hansen, S. Helveg, and A. K. Datye, Adv. Catal. 50, 77 (2006).

${ }^{8}$ S. Amelinckx, D. vanDyck, J. Van Landuyt, and G. Van Tendeloo, Handbook of Microscopy: Applications in Materials Science, Solid-State Physics and Chemistry (VCH, Weinheim, 1997).

${ }^{9}$ H. Permana, S. Lee, and K. Y. S. Ng, Catal. Lett. V24, 363 (1994).

${ }^{10}$ S. Helveg, J. V. Lauritsen, E. Laegsgaard, I. Stensgaard, J. K. Norskov, B. S. Clausen, H. Topsoe, and F. Besenbacher, Phys. Rev. Lett. 84, 951 (2000).

${ }^{11}$ M. Komiyama, K. Kiyohara, E. Yoda, T. Kubota, and Y. Okamoto, Jpn. J. Appl. Phys., Part 1 44, 5455 (2005).

${ }^{12}$ H. H. Rotermund, Surf. Sci. 283, 87 (1993).

${ }^{13}$ E. Bauer, J. Phys.: Condens. Matter 13, 11391 (2001).

${ }^{14}$ S. Gunther, B. Kaulich, L. Gregoratti, and M. Kiskinova, Prog. Surf. Sci. 70, 187 (2002).

${ }^{15}$ C. M. Schneider and G. Schönhense, Rep. Prog. Phys. 65, R1785 (2002).

${ }^{16}$ F. Esch, S. Gunther, E. Schutz, A. Schaak, I. Kevrekidis, M. Marsi, M. Kiskinova, and R. Imbihl, Catal. Lett. 52, 85 (1998).

${ }^{17}$ H. Marbach, M. Hinz, S. Gunther, L. Gregoratti, M. Kishkinova, and R. Imbihl, Chem. Phys. Lett. 364, 207 (2002).

${ }^{18}$ G. Beamson, H. Q. Porter, and D. W. Turner, Nature (London) 290, 556 (1981).

${ }^{19}$ B. P. Tonner, D. Dunham, T. Droubay, and M. Pauli, J. Electron Spectrosc. Relat. Phenom. 84, 211 (1997).

${ }^{20}$ M. Escher et al., J. Electron Spectrosc. Relat. Phenom. 144, 1179 (2005).

${ }^{21}$ Y. Yamaguchi, S. Takakusagi, Y. Sakai, M. Kato, K. Asakura, and Y. Iwasawa, J. Mol. Catal. A: Chem. 141, 129 (1999).

${ }^{22}$ K. Asakura, Catal. Surv. Asia 7, 177 (2003).

${ }^{23}$ H. Yasuhuku, Y. Ohminami, T. Tsutsumi, K. Asakura, M. Kato, Y. Sakai, Y. Kitajima, and Y. Iwasawa, Chem. Lett. 8, 842 (2002).

${ }^{24}$ H. Yasuhuku et al., J. Appl. Phys. 43, 7682 (2004).

${ }^{25}$ H. Spiecker et al., Nucl. Instrum. Methods Phys. Res. A 406, 499 (1998).

${ }^{26}$ G. K. L. Marx, V. Gerheim, and G. Schönhense, J. Electron Spectrosc. Relat. Phenom. 84, 251 (1997).

${ }^{27}$ K. Tsuno, Rev. Sci. Instrum. 64, 659 (1993).

${ }^{28}$ M. Kato and K. Tsuno, Nucl. Instrum. Methods Phys. Res. A A298, 296 (1990).

${ }^{29}$ K. Tsuno, Optik (Stutt) 89, 31 (1991).

${ }^{30}$ H. Niimi et al., Appl. Surf. Sci. 237, 641 (2004).

${ }^{31}$ H. Niimi et al., Appl. Surf. Sci. 241, 131 (2005).

${ }^{32}$ H. Rose, Optik (Stutt) 77, 26 (1987).

${ }^{33}$ M. Kato, Doctoral thesis, Tokyo University, 1997. 
${ }^{34}$ G. Martinez and K. Tsuno, Ultramicroscopy 93, 253 (2002).

${ }^{35} \mathrm{G}$. Martinez and K. Tsuno, Ultramicroscopy 100, 105 (2004).

${ }^{36}$ D. Ioanoviciu, K. Tsuno, and G. Martinez, Rev. Sci. Instrum. 75, 4434 (2004).

${ }^{37}$ K. Tsuno, D. Ioanoviciu, and G. Martinez, J. Microsc. 217, 205 (2005).

${ }^{38}$ P. W. Hawkes and E. Kasper, Principles of Electron Optics (Academic,
New York, 1989), Vol. 1.

${ }^{39} \mathrm{H}$. Niimi et al., Surf. Sci. (in press).

${ }^{40}$ T.-T. Tang, Optik (Stutt) 74, 51 (1986).

${ }^{41}$ M. R. Smith and E. Munro, Optik (Stutt) 74, 7 (1986).

${ }^{42}$ M. R. Scheinfein, Optik (Stutt) 82, 99 (1989).

${ }^{43}$ H. Steinbigler, Ph.D. thesis, Technisch Universitat München, 1969. 